\title{
Relative stabilities of two difluorodiazene isomers: density functional and molecular orbital studies
}

\author{
This article was published in the following Dove Press journal: \\ Reports in Theoretical Chemistry \\ 25 June 2012 \\ Number of times this article has been viewed
}

\section{Jibanananda Jana}

Department of Chemistry, Krishnagar Government College, Krishnagar, Nadia, West Bengal, India
Correspondence: Jibanananda Jana Department of Chemistry, Krishnagar Government College, Krishnagar, Nadia, West Bengal, India, PIN-74I I0I Email jiban_jana@yahoo.co.in

\begin{abstract}
The N-N bond length in the cis isomer of difluorodiazene is shorter than that in the trans isomer, giving the cis isomer higher stability. The energy partitioning approach identifies the necessary and dictating parameters responsible for the higher $\mathrm{N}-\mathrm{N}$ bond energy and higher non-bonded F-F interaction energy in the cis isomer. Using density functional theory, the cis isomer is found to have higher chemical hardness and lower softness, and hence, it has higher stability than the trans isomer on the basis of the principle of maximum hardness. Localized molecular orbital study shows that the cis isomer has a higher strength of delocalization of the lone pairs of electrons on the $F$ atoms than does the trans isomer, leading to higher stability of the cis isomer.
\end{abstract}

Keywords: cis/trans isomers, difluorodiazene, PMH, energy partitioning, localized molecular orbital, chemical hardness, softness

\section{Introduction}

It is well known ${ }^{1}$ that difluorodiazine (dinitrogen difluoride) $\mathrm{N}_{2} \mathrm{~F}_{2}$, is a gas with two isomers - cis and trans - which are shown in Figure 1. It is also known that the cis isomer predominates $(\sim 90 \%)$ at $25^{\circ} \mathrm{C}$ over the trans isomer.

It seems to be very obvious that the two fluorine atoms in the cis isomer, due to their greater proximity than in the trans isomer, undergo strong repulsion involving the lone pairs of electrons on the F atoms and that cis isomer is less stable than the trans one. However, the reverse observation, suggests the need to consider this probem from another angle to find a more fruitful solution.

There have been both theoretical and experimental studies regarding the relative stability of cis/trans isomers. ${ }^{2-13}$ Mouräo and Melo, ${ }^{2}$ using Mayer's energy decomposition method at the Hartree-Fock level, demonstrated that the main stabilizing effects of the cis isomer in the dihalo ethylenes are the energy terms associated with the interaction of halogen substituents with carbon atoms. With the help of the simple reversedphase column liquid chromatographic method, Ismail et $\mathrm{al}^{3}$ showed that the higher percentage composition of the monocrotophos cis isomer in the acetonitrile eluent indicates its higher stability. Concepción et $\mathrm{al}^{4}$ demonstrated the kinetic preferences of isomer formation. Hush et $\mathrm{al}^{5}$ reported the relative thermodynamic stabilities of cisand trans- $\left[\mathrm{PtCl}_{2}\left(\mathrm{H}_{2} \mathrm{O}\right)_{2}\right]$ in an aqueous solution using various methods and basis sets, concentrating largely on the role of solvation. Through gamma radiation, Alfaia et $\mathrm{al}^{7}$ indicated the higher susceptibility of the cis double bond to autoxidation compared to the trans configuration. Paul et $\mathrm{al}^{10}$ indicated the adjustability of the cis percentage and the conformational stability toward the intermolecular H-bonding effects. Schieber 

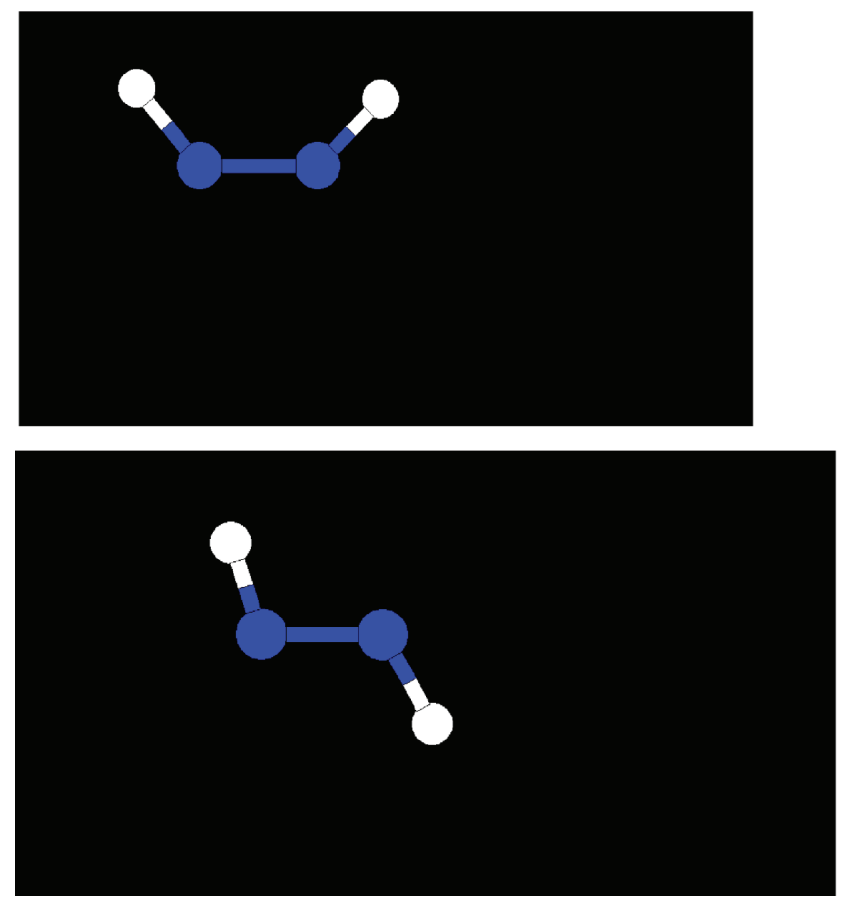

Figure I Optimized geometries of the cis and trans isomers of difluorodiazene $\left(\mathrm{N}_{2} \mathrm{~F}_{2}\right)$.

Notes: Cis $-\mathrm{N}_{2} \mathrm{~F}_{2}$; Blue balls: Nitrogen; White balls: Fluorine; Bond length $(\mathrm{N}-\mathrm{N})=1.239$; Bond lengths $(\mathrm{N}-\mathrm{F})=1.239$; Bond angle $(\angle \mathrm{FNN})=114.59$. Trans$\mathrm{N}_{2} \mathrm{~F}_{2}$; Blue balls: Nitrogen; White balls: Fluorine; Bond length $(\mathrm{N}-\mathrm{N})=1.24 \mathrm{I}$; Bond lengths $(\mathrm{N}-\mathrm{F})=1.239$; Bond angle $(\angle \mathrm{FNN})=109.93$. Bond lengths are in $\AA$ and bond angles are in degrees.

Abbreviations: $\angle \mathrm{FNN}$, angle between N-F and N-N bonds.

and Caele ${ }^{13}$ demonstrated that while trans configuration of carotenoids predominates, the cis isomer takes the prominent role for food processing under the alteration of provitamin activity, bioavailability, and antioxidant capacity.

The present study investigates whether there are any differences in the geometrical parameters between the two isomers, and if such differences do exist, the reasons behind them. Further investigation will study the role of global hardness on the relative stabilities of the two isomers as well as the reason behind the closer proximity of the two $F$ atoms. The study will also deal with whether there is any delocalization of the lone pairs of electrons on the $F$ atoms over the $\mathrm{F}-\mathrm{N}-\mathrm{N}-\mathrm{F}$ skeleton, and if this does happen, how it dictates the relative stabilities of the two isomers.

Figure 1 shows that the $\mathrm{N}-\mathrm{N}$ bond length in the cis isomer is shorter than that in the trans isomer, whereas the $\mathrm{N}-\mathrm{F}$ bonds in both isomers have the same bond length. Thus, the stability of the isomers is related to the $\mathrm{N}-\mathrm{N}$ bond strength. The related questions are as follows:

i. How does the $\mathrm{N}-\mathrm{N}$ bond strength render more stability to the cis isomer than the trans isomer and which parameter is responsible for the formation of the strong $\mathrm{N}-\mathrm{N}$ bond? ii. How do the highest occupied molecular orbital and lowest unoccupied molecular orbital (HOMO-LUMO) energy gaps and global hardnesses in the two isomers affect the stabilities of the molecules?

iii. What are the probable reasons for the closer proximity of the two $F$ atoms in the cis isomer? On the other hand, what is the nature of the interaction between the nonbonding $F$ atoms that makes them take the more stable vicinal position in the cis isomer?

iv. Does delocalization affect the electron pairs on the $F$ atoms with regard to the extra stability of the cis isomer? If so, what is the difference in the strength of the delocalization of the lone pairs of electrons on the $F$ atoms in the two cases?

It is necessary to invoke the density functional and $\mathrm{MO}$ theories to investigate the relative stabilities of the two isomers. The MO energy partitioning approach is to identify the parameters responsible for the $\mathrm{N}-\mathrm{N}$ bond strength and the nature of the interaction between non-bonding $F$ atoms in both isomers. Localized MO (LMO) study is used to investigate the nature and strength of the delocalization of the lone pairs of electrons on the $F$ atoms over the $\mathrm{F}-\mathrm{N}-\mathrm{N}-\mathrm{F}$ skeleton in the cis and trans isomers.

In the present study, I have investigated the reasons behind the unusual stability order of the two $\mathrm{N}_{2} \mathrm{~F}_{2}$ isomers. The work is organized as follows:

i. The HOMO-LUMO energy gap $(\Delta \varepsilon)$, global hardness $(\eta)$, and global softness $(S)$ for both the isomers are calculated to investigate the relative stabilities of the two $\mathrm{N}_{2} \mathrm{~F}_{2}$ isomers.

ii. The diatomic energy components for the $\mathrm{N}-\mathrm{N}$ bonded and $\mathrm{F}-\mathrm{F}$ non-bonded interaction energies in both isomers are calculated to identify the parameters necessary for the formation of the stronger $\mathrm{N}-\mathrm{N}$ bond and stronger non-bonded $\mathrm{F}-\mathrm{F}$ interaction in the cis isomer.

iii. The LMOs of both the isomers are calculated to investigate the role of delocalizing lone pairs of electrons on the $F$ atoms as well as their strength of delocalization with regard to the extra stability of the cis isomer over the trans one.

The conceptual density functional theory (DFT), a new branch of quantum mechanics, offers some new fundamental global parameters: chemical potential $(\mu),{ }^{14}$ chemical hardness $(\eta),{ }^{15}$ and its reverse concept global softness $(S),{ }^{15}$ of which global hardness and softness are of prime importance in the present work; $\mu$, which characterizes the escaping tendency of electrons, was discovered by Parr et $\mathrm{al}^{16}$ as a new, important property of chemical systems. Chemical hardness indicates 
the resistance of charge transfer. The higher value of $\eta$ makes a chemical species harder and indicates its lower chemical reactivity. Both $\eta$ and $\mu$ are successful in the rationalization of chemical processes. ${ }^{17-20} \mu, \eta$, and $S$ are new concepts of potential importance in chemistry and are established as fundamental quantities of molecular electronic structure and reactivity. ${ }^{21-25}$

In this regard, the principle of maximum hardness $(\mathrm{PMH})$, which was originally introduced by Pearson, ${ }^{22}$ is the one major contribution of DFT to structural and theoretical chemistry, leading to the characterization of reactants, products, and transition states of different kinds of chemical reactions. ${ }^{20,22,25-28}$ According to the $\mathrm{PMH}$, molecules tend to attain the state of maximum hardness when they are at equilibrium. Thus, when a system moves toward a more stable configuration, its global hardness increases and reaches its maximum when electronic energy reaches its minimum. This relationship between stability and hardness indicates that the PMH is a good descriptor of the global reactivity of a system. ${ }^{29}$

Rigorous and general proof of the $\mathrm{PMH}$ was given by Parr and Chattaraj et al, ${ }^{30,31}$ and it appears that the PMH is a fundamental, broadly applicable electronic structure rule. ${ }^{25}$ Although the $\mathrm{PMH}$ is demonstrated to be valid under the conditions of constant external potential $(v(r))$ and chemical potential, relaxation occurs in different systems. ${ }^{32,33}$ As any chemical reaction involves structural and electronic reordering, the external potential hardly remains constant. Morell et $\mathrm{al}^{34}$ defined a new index in terms of the variation of hardness with respect to the external potential. The $\mathrm{PMH}$ has been numerically tested in a number of calculations through the computation of global hardness for a variety of individual molecules during the process of structural inversion, deformation, and internal rotation in various types of simple reactions and in correlating the order of the stability and reactivity of structurally related molecules. ${ }^{27,28,35-46}$ The alteration of molecular polarizability with global hardness through the distortion of bond length was investigated by Roy et al. ${ }^{47}$

In MO theory, a molecule is nothing but a collection of nuclei and electronic charges are distributed through MOs that are fully delocalized and called canonical MOs (CMOs) or spectroscopic MOs (SMOs). ${ }^{48}$ Although CMOs are greatly helpful to spectroscopic studies, they remain silent about routine chemistry involving lone pairs and bond pairs, ie, they do not appear in the delocalized version of the quantum chemical method. It was discovered that a set of CMOs or SMOs is one of the many possible unitary bases in the Hartree-Fock space and the new sets of MOs, which conform to the chemical intuition in terms of lone pairs and bond pairs, can be generated by suitable unitary transformation in the same Hartree-Fock space. Such MOs are called LMOs.

In the present study, the CMOs of each molecular species are generated at their optimized geometries by the complete neglect of differential overlap (CNDO)/2 method of Pople et al. ${ }^{49}$ The CMOs are then transformed into LMOs using the method of Trindle and Sinanoğlu. ${ }^{50}$ Through the generation of the bond pairs and lone pairs of the isomers under investigation, the goal is to identify the nature and strength of the delocalization of the lone pairs on the $F$ atoms, and hence, to determine the relative stabilities of the isomers.

Using the energy partitioning method of Fischer and Kollmar, ${ }^{51}$ the total energy of a molecule can be partitioned into monoatomic and diatomic parts; thus, the reasons for the stronger $\mathrm{N}-\mathrm{N}$ bond formation in the cis isomer as well as the stable vicinal position of the two $F$ atoms in the cis isomer will be investigated by analyzing the diatomic interaction energy components.

\section{General definitions of density functional theory}

It should be noted that $N$ (the number of electrons) and $v(r)$ (the external potential) determine the perturbations as occurring in a chemical reaction. Thus, the reactivity of the molecules in terms of the system response to the variation of $N$ and $v(r)$ can be studied under the theoretical framework of DFT. ${ }^{52}$ The response to changes in the number of electrons when external potential remains constant is measured at the first order by $\mu$ and at the second order by $\eta$. The variation of the external potential without charge transfer is measured by the electron density $(\rho(r))$ at the first order and by the Fukui function $(f(r))$ at the second order. ${ }^{53}$ As the energy is a function of $N$ and $v(r)$, the first order variation in the total energy in terms of the simultaneous alteration of $N$ and $v(r)$ leads to the following expression:

$$
d E=(\partial E / \partial N)_{v(r)} d N+\int(\partial E / \partial v(r))_{N} d v(r) d r
$$

where

$$
\mu=(\partial E / \partial N)_{v(r)} \text { and } \rho(r)=(\partial E / \partial v(r))_{N}
$$

$\mu$ is a global property that characterizes the electron transfer associated with any chemical process and appears as the 
Lagrange multiplier with the condition that $\mathrm{p}(r)$ integrates to $N$. Now, the total differential of the chemical potential and electronic density ultimately goes to the following expressions:

$\eta=(\partial \mu / \partial N)_{v(r)}$ and $f(r)=(\partial \rho(r) / \partial N)_{v(r)}=[\delta \mu / \delta v(r)]_{N}$

Hardness can be described as the resistance to charge transfer.

\section{Operational definitions}

From the rigorous density functional definition, an exact calculation is not feasible. Operational definitions of the parameters are needed. Global hardness is obtained as

$$
\eta=(I-A) / 2
$$

where $I$ and $A$ are the ionization potential and electron affinity, respectively, can be replaced by the energy of the HOMO and $L U M O$, respectively, using Koopmans' theorem, ${ }^{54}$ and are given as follows:

$$
I=-\mathcal{E}_{\text {номо }} ; \quad A=-\mathcal{E}_{\text {LUмо }}
$$

As one can arrive at the DFT-based concepts in terms of semi-empirical methods ${ }^{14}$ (eg, NDO) and because conclusions of DFT can be effectively tested by semi-empirical theories, ${ }^{22,23,28,35-37}$ in this investigation, the CNDO/2 method of Pople et $\mathrm{al}^{49}$ was used, taking STO as the basis set. S, the global softness, is the inverse concept of global hardness and is defined as follows:

$$
S=1 / \eta
$$

For the present purposes, the operational definition of chemical potential is not required. Thus, its operational definition is avoided here.

\section{Energy partitioning of Fischer and Kollmar}

Pople et $\mathrm{al}^{55}$ first noted the possibility of partitioning the total molecular energy $(E)$ obtained with the semi-empirical CNDO theory into one and two center terms:

$$
E=\sum_{A} E_{A}+\sum_{A>B} \sum_{B} E_{A B}
$$

A more detailed analysis of the two center terms was done by Gordon ${ }^{56}$ to explain the torsional barriers. For the present purposes, the matters related to the one center term will be set aside and the explicit formulae of only two center terms will be used. However, $\mathrm{E}_{\mathrm{A}}$ and $\mathrm{E}_{A B}$ can be written as follows:

$$
\begin{aligned}
& E_{A}=E_{A}{ }^{U}+E_{A}{ }^{J}+E_{A}{ }^{K} \text { and } \\
& E_{A B}=E_{A B}{ }^{R}+E_{A B}{ }^{V}+E_{A B}{ }^{J}+E_{A B}{ }^{K}+E_{A B}{ }^{N} .
\end{aligned}
$$

Superscripts characterize the physical nature of the energy terms and subscripts specify the atoms. $E_{A B}{ }^{V}=$ the potential energy of the electrons on atom $A$ in the field of nucleus $B$ plus that of the electrons on atom $B$ in the field of nucleus $A$ and is expressed as follows:

$$
E_{A B}^{V}=-P_{A} V_{A B}-p_{B} V_{B A},
$$

where $P_{A}=\sum_{\mu \in A} P_{\mu \mu}$ and $\mathrm{P}_{\mu \mu}$ are the elements of the bond order matrix with atomic orbital $\mu$ on atom $A$. Similarly, $p_{B}=\sum_{\nu \in B} p_{v v}$ and $p_{v v}$ are the elements of the bond order matrix with atomic orbital $v$ on atom $B . V_{A B}$ is the potential energy of an electron on atom $A$ in the field of nucleus $B$, and $V_{B A}$ is the potential energy of an electron on atom $B$ in the field of nucleus $A$.

$E_{A B}{ }^{R}$ is the contribution of resonance integrals to the energy of the $A-B$ bond and is the main feature of the covalent bond and is expressed as follows:

$$
E_{A B}^{R}=2 \sum_{\mu \in A} \sum_{\nu \in B} p_{\mu \nu} \beta_{\mu v} S_{\mu \nu}
$$

where $\beta_{\mu \nu}$ is a parameter that is dependent on orbitals $\mu$ and $v$. $S_{\mu v}$ is the overlap of the STOs $\mu$ and $\nu$.

$E_{A B}{ }^{J}$ accounts for the repulsion of the electrons on the atoms $A$ and $B$ and is expressed as follows:

$$
E_{A B}^{J}=P_{A} P_{B} \gamma_{A B}
$$

where $\gamma_{A B}$ is the electronic repulsion between an electron on atom $A$ and an electron on atom $B$.

$E_{A B}{ }^{\mathrm{K}}$ accounts for the electronic exchange interactions and is expressed as follows:

$$
E_{A B}^{K}=-1 / 2 \gamma_{A B} \sum_{\mu \in A} \sum_{\nu \in B} P_{\mu \nu}^{2}
$$

$E_{A B}{ }^{N}$ is the nuclear repulsion energy of the nuclei $A$ and $B$ and is expressed as follows:

$$
E_{A B}^{N}=\frac{Z_{A} Z_{B}}{R_{A B}},
$$

where $Z_{A}$ and $Z_{B}$ are the nuclear charges on $A$ and $B$ respectively and $R_{A B}$ is the distance between the nuclei. 


\section{Localized molecular orbitals \\ from the method of Trindle and Sinanoĝlu}

Here, the definition of the localization measure, $D$, is based on the idea that an electron in a local orbital should interact maximally with the electron sharing the same orbital. For a given set of doubly occupied orbitals, $\left(\phi_{1}, \ldots \ldots \ldots, \phi_{n}\right), D$ is defined below as the sum of orbital coulomb energies:

$$
\begin{aligned}
D_{N} & =\sum_{n=1}^{N}\left[\phi_{n}{ }^{2} / \phi_{n}{ }^{2}\right] \\
& =\sum_{n=1}^{N} \iint \phi_{n}{ }^{2}{ }_{(1)} r_{12}^{-1} \phi_{n}{ }^{2}{ }_{(2)} d \tau_{1} d \tau_{2}
\end{aligned}
$$

$D$ will be a maximum for maximally localized orbitals. A simple iterative technique was proposed by Edmiston and Reudenberg ${ }^{57}$ for transforming the orbitals to the set that maximized $D$. They considered the exactly soluble two-orbital case, molecular orbitals eg, $\phi_{\mathrm{i}}$ and $\phi_{\mathrm{j}}$ are ones of many possible unitary transformations and through unitary transformation, another set of molecular orbitals is generated eg, $u_{i}$ and $u_{j}$, which are more localized. Now the quest is for that unitary transformation which is more localized occupied molecular orbitals. That is, what is $\sin \alpha_{\mathrm{ij}}$ and $\cos \alpha_{\mathrm{ij}}$ in Equation (2)?

$$
\left|\begin{array}{rr}
\cos \alpha_{i j} & -\sin \alpha_{i j} \\
\sin \alpha_{i j} & \cos \alpha_{i j}
\end{array}\right|\left|\begin{array}{c}
\phi_{i} \\
\phi_{j}
\end{array}\right|=\left|\begin{array}{l}
u_{i} \\
u_{j}
\end{array}\right|
$$

Maximization of $D_{2}$ with respect to the angle $\alpha_{i j}$ leads to the expressions in Equation (3).

$$
\begin{aligned}
& \cos 4 \alpha_{i j}=-A_{i j} /\left[A_{i j}^{2}+B_{i j}^{2}\right]^{1 / 2} \\
& \sin 4 \alpha_{i j}=B_{i j} /\left[A_{i j}^{2}+B_{i j}^{2}\right]^{1 / 2} \\
& A_{i j}=\left[\phi_{i} \phi_{j} / \phi_{i} \phi_{j}\right]-\frac{1}{4}\left[\phi_{i}^{2}-\phi_{j}^{2} / \phi_{i}^{2}-\phi_{j}^{2}\right] \\
& B_{i j}=\left[\phi_{i}^{2}-\phi_{j}^{2} / \phi_{i} \phi_{j}\right]
\end{aligned}
$$

The assumptions of the $\mathrm{CNDO} / 2$ method on the two electron integrals allows for considerable simplification of the Edmiston-Ruedenberg transformation, leading to Equation (4), which drastically reduces the time required per iteration.

$$
\begin{aligned}
A_{i j} & =\sum_{r t}[r r / t t]\left\{\left(a_{i r}^{2}-a_{j r}^{2}\right) a_{i t} a_{j t}-\frac{1}{4}\left(a_{i r}^{2}-a_{j r}^{2}\right)\left(a_{j t}^{2}-a_{i t}^{2}\right)\right\} \\
B_{i j} & =\sum_{r t}[r r / t t]\left\{\left(a_{i r}^{2}-a_{j r}^{2}\right) a_{i t} a_{j t}\right\}
\end{aligned}
$$

\section{Results and discussion}

Figure 1 shows the optimized geometrical parameters of both the cis and trans isomers of difluorodiazene. This figure shows that the $\mathrm{N}-\mathrm{N}$ bond length in the cis isomer is shorter than that in the trans isomer, although the $\mathrm{N}-\mathrm{F}$ bond length in both cases is identical. Thus, the difference in the $\mathrm{N}-\mathrm{N}$ bond strength seems to be the reason behind the extra stability of the cis isomer. Table 1 shows the various energy components of the $\mathrm{N}-\mathrm{N}$ bond energy for both isomers. Table 1 demonstrates that the numerical values of $E_{N-N}{ }^{J}-$ the energy of repulsion among the electrons on one $N$ atom with those on another $N$ atom - and $E_{N-N}{ }^{N}$ - the nuclear repulsion energy of the two $N$ nuclei - of the cis isomer are greater than those of the trans isomer. Thus, the greater $E_{N-N}{ }^{J}$ and $\mathrm{E}_{\mathrm{N}-\mathrm{N}}{ }^{\mathrm{N}}$ values of the cis isomer tend to reduce the $\mathrm{N}-\mathrm{N}$ bond strength, but all other diatomic parameters $-E_{N-N}{ }^{V}$, the potential energy of the electrons on one $N$ atom in the field of the nucleus of another $N$ atom and vice versa; $E_{N-N}{ }^{K}$, electronic exchange interactions; and $E_{N-N}{ }^{R}$, the contribution of resonance integrals to the energy of the $\mathrm{N}-\mathrm{N}$ bond - strengthen the aforesaid bond in the cis isomer; the most contributing parameter is $E_{N-N}$. Thus, the electron-nuclear attraction potential in the $\mathrm{N}-\mathrm{N}$ bond takes the lead for the formation of the stronger $\mathrm{N}-\mathrm{N}$ bond in the cis isomer. Table 4 shows that the difference in $E^{V}$ of the two isomers corresponding to the $\mathrm{N}-\mathrm{N}$ bonded interaction is 20.22 times more than the total energy difference, $\Delta E$, whereas the differences in $E^{K}$ and $E^{R}$ of the two isomers are 0.61 and 2.52 times, more than the $\Delta E$, respectively. From the above comparative result, $E_{N-N} V$ is found to be the controlling parameter of the large stability of the cis isomer.

Further investigation will determine the reason for the closer proximity of the two $F$ atoms in the cis isomer compared those in the trans isomer. Table 2 shows the various energy compared to components for the non-bonded $\mathrm{F}-\mathrm{F}$ diatomic interaction energy. Table 2 demonstrates that while $E_{F-F}^{J}$ and $E_{F-F}^{N}$ lower the $\mathrm{F}-\mathrm{F}$ interaction strength in the cis isomer, $E_{F-F}, E_{F-F}^{K}$, and $E_{F-F}^{R}$

Table I N-N bond energy components and total $\mathrm{N}-\mathrm{N}$ bond energy $\left(E_{\mathrm{N}-\mathrm{N}}\right)$ of the cis and trans isomers of difluorodiazene, $\mathrm{N}_{2} \mathrm{~F}_{2}$

\begin{tabular}{lllllll}
\hline Isomers & $\begin{array}{l}E_{N-N}{ }^{j} \\
(\mathrm{au})\end{array}$ & $\begin{array}{l}E_{N-N}{ }^{N} \\
(\mathrm{au})\end{array}$ & $\begin{array}{l}E_{N-N}{ }^{N} \\
(\mathrm{au})\end{array}$ & $\begin{array}{l}E_{N-N}{ }^{K} \\
(\mathrm{au})\end{array}$ & $\begin{array}{l}E_{N-N}{ }^{2} \\
(\mathrm{au})\end{array}$ & $\begin{array}{l}E_{N-N} \\
(\mathrm{au})\end{array}$ \\
\hline $\mathrm{Cis} \mathrm{N}_{2} \mathrm{~F}_{2}$ & 9.79794 & 10.67745 & -20.01785 & -0.39094 & -1.65850 & -1.59190 \\
Trans- $\mathrm{N}_{2} \mathrm{~F}_{2}$ & 9.76283 & 10.65889 & -19.96811 & -0.38944 & -1.65231 & -1.58814 \\
\hline
\end{tabular}

Note: Energy values are in au. 
Table $2 \mathrm{~F}-\mathrm{F}$ non-bonded energy components and total $\mathrm{F}-\mathrm{F}$ non-bonded interaction energy $\left(E_{F-F}\right)$ of the cis and trans isomers of difluorodiazene, $\mathrm{N}_{2} \mathrm{~F}_{2}$

\begin{tabular}{|c|c|c|c|c|c|c|}
\hline Isomers & $\begin{array}{l}E_{F-F}^{J} \\
\text { (au) }\end{array}$ & $\begin{array}{l}E_{F-F}{ }^{N} \\
\text { (au) }\end{array}$ & $\begin{array}{l}E_{F-F}^{v} \\
\text { (au) }\end{array}$ & $\begin{array}{l}E_{F-F}{ }^{K} \\
\text { (au) }\end{array}$ & $\begin{array}{l}E_{F-F}{ }^{R} \\
\text { (au) }\end{array}$ & $\begin{array}{l}E_{F-F} \\
\text { (au) }\end{array}$ \\
\hline Cis- $\mathrm{N}_{2} \mathrm{~F}_{2}$ & 11.76866 & II.42220 & -23.18816 & -0.00233 & -0.00275 & -0.00238 \\
\hline Trans- $\mathrm{N}_{2} \mathrm{~F}_{2}$ & 8.55705 & 8.29248 & -16.84745 & -0.00206 & -0.0005 I & -0.00049 \\
\hline
\end{tabular}

Note: Energy values are in au.

are responsible for the stronger $\mathrm{F}-\mathrm{F}$ interaction, in which $E_{F-F}$ plays the primary role. Thus, the variation trends of the diatomic energy components for the F-F non-bonded interaction energy mimic those for the bonded $\mathrm{N}-\mathrm{N}$ energy.

Table 4 reveals that the difference in $E^{V}$ of the two isomers corresponding to the $F-\mathrm{F}$ non-bonded interaction is 2577.5 times greater than $\Delta \mathrm{E}$ whereas the differences in $E^{K}$ and $E^{R}$ of the two isomers are 0.11 and 0.91 times more than the $\Delta E$ respectively. Thus, the comparative study reveals that the dictating parameter of the large stability of the cis isomer is $E_{F-F}{ }^{V}$. Further comparison involving the contributing parameters of the $\mathrm{N}-\mathrm{N}$ bonded and $\mathrm{F}-\mathrm{F}$ non-bonded interaction energy shows that the most dictating parameter for the larger stability of the $\mathrm{N}_{2} \mathrm{~F}_{2}$ cis isomer compared to the trans isomer is $E_{F-F}$.

Table 3 shows the energy of $H O M O\left(\varepsilon_{\text {номо }}\right)$, the energy of $\operatorname{LUMO}\left(\varepsilon_{L U M O}\right), \Delta \varepsilon, \eta, \mathrm{S}$, and the total energy of both isomers. From Table 3, it can be seen that the numerical value of $\eta$ in the cis isomer is $0.33681 \mathrm{au}$ and $0.33474 \mathrm{au}$ in the trans isomer. The cis isomer has a total energy of $-78.90530 \mathrm{au}$ and the trans isomer has a total energy of $-78.90284 \mathrm{au}$. An analysis of these values for both isomers shows that the more stable cis isomer has a higher $\eta$ value than the less stable trans isomer. Thus, the above observation is in nice conformity with the PMH.

The relative capabilities of delocalization of lone pair of electrons on $\mathrm{F}$ atoms over the F-N-N-F skeleton in both the cis and trans isomers will be investigated. It should be noted that any unitary transformation of MOs leaves the total energy of the system intact. The chemists' routine chemistry usually deals with the lone pairs and bond pairs for the at a glance view on the chemical system for the explanation of a behavior. In delocalized molecular orbitals, the concept of lone pairs and bond pairs vanishes. But localized molecular orbitals generate those and thus their routine chemistry is preserved. In the present study, based on the knowledge of the nature of lone pairs, their delocalization strength is easy to detect, which is related to the contribution to the stability through the lowering of kinetic energy pressure ${ }^{58}$ and also from the view point of particle in a 3D box model.

Table 5 shows the LMOs of the cis isomer. Table 5 demonstrates that LMO 21.p.(1F), the second lone pair on the first $F$ atom, and LMO 31.p.(2F), the third lone pair on the second $F$ atom, become delocalized over the $\mathrm{F}-\mathrm{N}-\mathrm{N}-\mathrm{F}$ skeleton to diminish the kinetic energy pressure from those electron pairs, leading to their strong contribution toward the overall stability of the cis isomer. Table 6 displays the LMOs of the trans isomer. From Table 6, it can be seen that LMO 11.p.(1F), the first lone pair on the first $F$ atom, and LMO 31.p.(2F), the third lone pair on the second $F$ atom, become delocalized over the $\mathrm{F}-\mathrm{N}-\mathrm{N}-\mathrm{F}$ skeleton. Thus, in the trans isomer, like in the cis isomer, the third lone pair on the second $F$ atom participates in the delocalization over the F-N-N-F skeleton; however, unlike the cis isomer, the first lone pair on the first $F$ atom in the trans isomer takes the role of delocalization over the previous framework. Table 5 shows that the coefficient of $2 \mathrm{p}_{\mathrm{z}}$ orbital on the first $F$ atom for the formation of LMO 21.p.(1F) and the coefficient of the $2 \mathrm{p}_{\mathrm{Z}}$ orbital on the second $F$ atom for the formation of LMO 31.p.(2F) have the same value (-0.9850). In LMO 21.p.(1F), the $2 \mathrm{p}_{\mathrm{z}}$ orbital on the first $F$ atom is in $\pi$-type interaction with the

Table 3 Energy of HOMO, $\varepsilon_{\text {номо }}(\mathrm{au})$; energy of $L U M O, \varepsilon_{\text {LUмо }}(\mathrm{au})$; global hardness, $\eta(\mathrm{au})$; global softness, $S$ (au); and total energy, $E$ (au), of the cis and trans isomers of difluorodiazene, $\mathrm{N}_{2} \mathrm{~F}_{2}$

\begin{tabular}{|c|c|c|c|c|c|c|}
\hline Isomers & $\begin{array}{l}\text { Energy of HOMO } \\
\varepsilon_{\text {номо }} \\
\text { (au) }\end{array}$ & $\begin{array}{l}\text { Energy of LUMO } \\
\varepsilon_{\text {LUMO }} \\
\text { (au) }\end{array}$ & $\begin{array}{l}\text { HOMO-LUMO } \\
\text { energy gap } \\
\Delta \varepsilon \\
\text { (au) }\end{array}$ & $\begin{array}{l}\text { Global } \\
\text { hardness } \\
\eta \\
\text { (au) }\end{array}$ & $\begin{array}{l}\text { Global } \\
\text { softness } \\
\text { S } \\
\text { (au) }\end{array}$ & $\begin{array}{l}\text { Total energy } \\
\text { E } \\
\text { (au) }\end{array}$ \\
\hline Cis- $\mathrm{N}_{2} \mathrm{~F}_{2}$ & $-0.567 \mid 4$ & 0.10648 & 0.67362 & 0.33681 & 2.96903 & -78.90530 \\
\hline Trans- $\mathrm{N}_{2} \mathrm{~F}_{2}$ & $-0.5667 \mid$ & 0.10276 & 0.66947 & 0.33474 & 2.98739 & -78.90284 \\
\hline
\end{tabular}

Abbreviations: HOMO, highest occupied molecular orbital; LUMO, lowest unoccupied molecular orbital. 
Table 4 Difference in the energy parameters $\left(E^{V}, E^{K}\right.$, and $\left.E^{R}\right)$ for both the $\mathrm{N}-\mathrm{N}$ bonded and $\mathrm{F}-\mathrm{F}$ non-bonded interactions and difference in total energy $(\Delta E)$ of the cis and trans isomers of difluorodiazene, $\mathrm{N}_{2} \mathrm{~F}_{2}$

\begin{tabular}{lllll}
\hline Interaction types & $\Delta E^{V}(\mathrm{au})$ & $\Delta E^{\mathrm{K}}(\mathrm{au})$ & $\Delta E^{R}(\mathrm{au})$ & $\Delta E(\mathrm{au})$ \\
\hline $\mathrm{N}-\mathrm{N}$ bonded & 0.04974 & 0.00150 & 0.00619 & 0.00246 \\
F-F non-bonded & $6.3407 \mathrm{I}$ & 0.00027 & 0.00224 & \\
\hline
\end{tabular}

Note: Energy values are in au.

$2 \mathrm{p}_{\mathrm{z}}$ orbitals on the second $F$, first $N$, and second $N$. Similarly, in LMO 31.p.(2F), the $2 \mathrm{p}_{\mathrm{Z}}$ orbital on the second $F$ atom is in $\pi$-type interaction with the $2 \mathrm{p}_{\mathrm{z}}$ orbital on the first $F$, first $N$, and second $N$. Further, Table 6 shows that the coefficient of the $2 \mathrm{p}_{\mathrm{z}}$ orbital on the first $F$ atom for the formation of LMO 11.p.(1F) and the coefficient of the $2 \mathrm{p}_{\mathrm{z}}$ orbital on the second $F$ atom for the formation of LMO 31.p.(2F) have the same value (-0.9858). In the formation of the previously mentioned LMOs of the trans isomer, like the cis isomer, the $2 \mathrm{p}_{\mathrm{z}}$ orbital on one $F$ atom is in $\pi$-type interaction with the $2 \mathrm{p}_{z}$ orbital on the other $F$ atom and both the $N$ atoms.

A comparison of Tables 5 and 6 shows that in Table 5, the second lone pair on the first $F$ atom in LMO 21.p.(1F) and the third lone pair on the second $F$ atom in LMO 31.p.(2F) of the cis isomer are delocalized to a greater extent than in the trans isomer, whose LMOs are shown in Table 6. Table 6 shows the greater contributions of the $2 \mathrm{p}_{\mathrm{z}}$ atomic orbitals on $1 \mathrm{~F}$ and $2 \mathrm{~F}$ for the formation of LMOs 11.p.(1F) and 31.p.(2F), respectively, which indicates the higher charge accumulation on those $F$ atoms compared to the population on the $F$ atoms in the cis isomer. There is then greater reluctance of those lone pairs on the $F$ atoms in the trans isomer toward delocalization. Thus, the lower delocalization abilities of those lone pairs on the $F$ atoms in the trans isomer make it less stable. Further comparison of Tables 5 and 6 shows that the coefficients of the $2 \mathrm{P}_{\mathrm{z}}$ orbitals on the $N$ atoms and $F$ atom (other than the most contributing one) toward LMO 31.p.(2F) and 11.p.(1F) formation in the trans isomer (Table 6) are smaller than those toward the formation of LMOs 21.p.(1F) and 31.p.(2F) in the cis isomer, indicating a higher charge density on the $N$ atoms in the cis form, and hence, a higher delocalization capacity of the lone pairs of electrons on the $F$ atoms in the cis isomeric form.

Thus, from the above LMO study on both isomers, it is very much expected that the higher delocalization strengths of the delocalizing lone pairs on the $F$ atoms in the cis isomer make the cis isomer relatively more stable than the trans isomer by lowering the kinetic energy pressure ${ }^{72}$ from those lone pairs, and also from the view point of particle in a 3D box model.

\section{Conclusion}

Figure 1 shows that the $\mathrm{N}-\mathrm{N}$ bond length in the cis isomer of $\mathrm{N}_{2} \mathrm{~F}_{2}$ is shorter than that in the trans isomer, and this difference in geometrical parameter leads to the higher stability of the cis isomer, which is nicely reflected in the DFT study. In conformity with the $\mathrm{PMH}$, it is found that the more stable cis isomer has higher global hardness and lower global softness than the trans isomer.

Using CNDO/2 energy partitioning, it is found that the electron-nuclear attraction potential $\left(E_{N-N}\right)$ involving the electron on one $N$ with the nucleus of another $N$, and vice

Table 5 Localized molecular orbitals (LMOs) of cis-difluorodiazene, $\mathrm{N}_{2} \mathrm{~F}_{2}$

\begin{tabular}{|c|c|c|c|c|c|c|c|c|c|c|c|c|}
\hline $\begin{array}{l}\text { LMOs/ } \\
\text { Atomic } \\
\text { orbitals }\end{array}$ & II.p.(IN) & $\sigma(I N-I F)$ & II.p.(IF) & $\sigma(\mathbf{N}-\mathbf{N})$ & II.p.(2F) & $\pi(\mathbf{N}-\mathbf{N})$ & $\sigma(2 \mathrm{~N}-2 \mathrm{~F})$ & 2I.p.(IF) & I.p.(2N) & 3I.p.(IF) & 2l.p.(2F) & 31.p.(2F) \\
\hline $\mathrm{IN}_{2 \mathrm{~S}}$ & -0.7715 & 0.2526 & 0 & $0.355 I$ & -0.0215 & 0 & 0.0323 & 0 & 0.0285 & $-0.028 \mathrm{I}$ & 0.0025 & 0 \\
\hline$I_{2 p x}$ & 0.1676 & -0.4076 & -0.0001 & 0.5304 & -0.0372 & 0 & $-0.037 \mid$ & 0 & $-0.047 \mid$ & -0.0427 & -0.0036 & 0 \\
\hline$I_{2 p y}$ & 0.6082 & 0.4260 & -0.0009 & $0.304 I$ & -0.0171 & 0 & 0.0203 & 0 & 0.0488 & -0.0233 & $0.004 I$ & 0 \\
\hline$I_{2 p z}$ & 0 & 0 & 0 & 0 & 0 & 0.7069 & 0 & -0.1329 & 0 & 0 & 0 & 0.1086 \\
\hline $2 \mathrm{~N}_{25}$ & 0.0283 & 0.0324 & 0.0023 & 0.3551 & $0.028 I$ & 0 & 0.2526 & 0 & -0.7715 & 0.0215 & 0 & 0 \\
\hline $2 N_{2 p x}$ & -0.0014 & 0.0120 & -0.0004 & -0.5901 & -0.0466 & 0 & -0.0131 & 0 & -0.5486 & -0.0384 & 0.0006 & 0 \\
\hline $2 \mathrm{~N}_{2 \mathrm{py}}$ & 0.0678 & 0.0406 & 0.0056 & -0.1601 & -0.0136 & 0 & 0.5894 & 0 & 0.3115 & -0.0142 & -0.0006 & 0 \\
\hline $2 \mathrm{~N}_{2 p z}$ & 0 & 0 & 0 & 0 & 0 & 0.7069 & 0 & 0.1086 & 0 & 0 & 0 & -0.1329 \\
\hline $\mathrm{IF}_{2 \mathrm{~S}}$ & -0.0005 & 0.3303 & -0.8962 & 0 & -0.0012 & 0 & -0.0174 & 0 & -0.0159 & 0.0998 & -0.0018 & 0 \\
\hline$I F_{2 p x}$ & -0.0010 & 0.5146 & 0.2469 & -0.0059 & 0 & 0 & -0.0286 & 0 & -0.0249 & -0.6949 & -0.0028 & 0 \\
\hline $\mathrm{IF}_{2 \mathrm{py}}$ & & -0.4583 & -0.3685 & -0.0043 & 0.0034 & 0 & 0.0251 & 0 & 0.0223 & -0.7084 & 0.0025 & 0 \\
\hline $\mathrm{IF}_{2 \mathrm{pz}}$ & 0 & 0 & 0 & 0 & 0 & -0.0171 & 0 & -0.9850 & 0 & 0 & 0 & -0.0146 \\
\hline $2 \mathrm{~F}_{2 \mathrm{~S}}$ & -0.0162 & -0.0174 & -0.0018 & 0 & -0.0996 & 0 & 0.3304 & 0 & -0.0005 & 0.0013 & -0.8962 & 0 \\
\hline $2 F_{2 p x}$ & 0.0018 & 0.0024 & 0.0001 & 0.0072 & -0.9923 & 0 & -0.0396 & 0 & 0 & 0.0025 & 0.0858 & 0 \\
\hline $2 \mathrm{~F}_{2 \mathrm{py}}$ & 0.0334 & 0.0379 & 0.0037 & 0.0012 & 0.0095 & 0 & -0.6879 & 0 & 0.0014 & -0.0023 & -0.4352 & 0 \\
\hline $2 F_{2 p z}$ & 0 & 0 & 0 & 0 & 0 & -0.0171 & 0 & -0.0146 & 0 & 0 & 0 & -0.9850 \\
\hline
\end{tabular}


Table 6 Localized molecular orbitals (LMOs) of trans-difluorodiazene, $\mathrm{N}_{2} \mathrm{~F}_{2}$

\begin{tabular}{|c|c|c|c|c|c|c|c|c|c|c|c|c|}
\hline $\begin{array}{l}\text { LMOs } \\
\text { Atomi } \\
\text { orbital }\end{array}$ & $\sigma(\mathbf{N}-\mathbf{N})$ & II.p.(IN) & II.p.(2N) & II.p.(2F) & $\sigma(I N-I F)$ & 2I.p.(2F) & 3I.p.(2F) & II.p.(IF) & 2I.p.(IF) & 31.p.(IF) & $\sigma(2 \mathrm{~N}-2 \mathrm{~F})$ & $\pi(\mathbf{N}-\mathbf{N})$ \\
\hline $\mathrm{IN}_{2 \mathrm{~S}}$ & 0.3508 & -0.7752 & 0.0201 & 0.0293 & -0.2495 & -0.0008 & 0 & 0 & -0.0004 & 0.027 I & -0.0306 & 0 \\
\hline $1 \mathrm{~N}_{2 p x}$ & 0.1219 & 0.5257 & 0.0428 & 0.0228 & -0.5303 & -0.0021 & 0 & 0 & -0.0005 & 0.0074 & -0.0383 & 0 \\
\hline $1 \mathrm{~N}_{2 \mathrm{py}}$ & 0.6017 & 0.3454 & -0.0204 & 0.0308 & 0.2563 & 0.0018 & 0 & 0 & -0.0006 & 0.0462 & 0.0277 & 0 \\
\hline$I_{2 p z}$ & 0 & 0 & 0 & 0 & 0 & 0 & 0.1063 & -0.1292 & 0 & 0 & 0 & -0.7069 \\
\hline $2 \mathrm{~N}_{2 \mathrm{~S}}$ & 0.3508 & -0.0200 & 0.7752 & -0.0269 & 0.0306 & -0.0004 & 0 & 0 & -0.0008 & -0.0292 & 0.2495 & 0 \\
\hline $2 N_{2 p x}$ & -0.1220 & 0.0430 & 0.5258 & 0.0075 & -0.0386 & 0.0005 & 0 & 0 & 0.0023 & 0.0228 & -0.5302 & 0 \\
\hline $2 N_{2 p y}$ & -0.6016 & -0.0204 & 0.3454 & 0.0463 & 0.0278 & 0.0007 & 0 & 0 & -0.0017 & 0.0308 & 0.2564 & 0 \\
\hline $2 N_{2 p z}$ & 0 & 0 & 0 & 0 & 0 & 0 & -0.1292 & 0.1063 & 0 & 0 & 0 & -0.7069 \\
\hline $\mathrm{IF}_{2 \mathrm{~S}}$ & 0.0011 & -0.0004 & -0.0113 & -0.0049 & -0.3313 & 0.0008 & 0 & 0 & -0.8936 & -0.1188 & 0.0193 & 0 \\
\hline$I F_{2 p x}$ & -0.0038 & 0.0009 & 0.0203 & 0.0079 & 0.5940 & -0.0015 & 0 & 0 & -0.4366 & 0.4537 & -0.0348 & 0 \\
\hline$I F_{2 p y}$ & -0.0054 & -0.0006 & -0.0119 & -0.0074 & -0.3506 & 0.0009 & 0 & 0 & 0.1044 & $0.880 \mathrm{I}$ & 0.0233 & 0 \\
\hline $\mathrm{IF}_{2 \mathrm{pz}}$ & 0 & 0 & 0 & 0 & 0 & 0 & -0.0139 & -0.9858 & 0 & 0 & 0 & 0.0162 \\
\hline $2 \mathrm{~F}_{2 \mathrm{~S}}$ & 0.0012 & 0.0114 & 0.0004 & 0.1196 & -0.0193 & -0.8934 & 0 & 0 & 0.0008 & 0.0048 & 0.3313 & 0 \\
\hline $2 F_{2 p x}$ & 0.0038 & 0.0202 & 0.0007 & 0.4533 & -0.0346 & 0.4370 & 0 & 0 & 0.0014 & 0.0079 & 0.5940 & 0 \\
\hline $2 F_{2 p y}$ & 0.0054 & -0.0117 & -0.0008 & 0.8802 & 0.0232 & -0.1036 & 0 & 0 & -0.0009 & -0.0074 & -0.3506 & 0 \\
\hline $2 F_{2 p z}$ & 0 & 0 & 0 & 0 & 0 & 0 & -0.9858 & -0.0139 & 0 & 0 & 0 & 0.0162 \\
\hline
\end{tabular}

versa, is greater in the cis isomer compared to the trans isomer, leading to a higher $\mathrm{N}-\mathrm{N}$ bond energy in the cis isomer. The exchange energy $\left(E_{N-N}{ }^{K}\right)$ and resonance energy $\left(E_{N-N}^{R}\right)$ vary in the same pattern as that of $\left(E_{N-N}^{V}\right)$ though variation of $\left(E_{N-N}^{J}\right)$ and $\left(E_{N-N}^{N}\right)$ run in the opposite direction and tend to decrease the $\mathrm{N}-\mathrm{N}$ bond energy in the cis isomer.

Further, an energy partitioning study on the non-bonded $\mathrm{F}-\mathrm{F}$ interaction energy was undertaken to identify the reason for the closer proximity of the two $F$ atoms in the cis isomer. The study shows that like the $\mathrm{N}-\mathrm{N}$ bond energy partitioning, $E_{F-F}{ }^{V}, E_{F-F}^{K}$, and $E_{F-F}^{R}$ tend to increase the interaction energy when $E_{F-F} V$ takes the lead and the opposing parameters, $E_{F-F}{ }^{J}$ and $E_{F-F}^{N}$, are overcome by the results of the former parameters.

The LMO study shows that the second lone pair on the first $F$ atom and the third lone pair on the second $F$ atom in the cis isomer become delocalized in a larger amount than the third lone pair on the second $F$ atom and the first lone pair on the first $F$ atom in the trans isomer. Thus, the lower kinetic energy pressure due to the higher delocalization capabilities of the lone pairs in the cis isomer is expected to render higher stability to the cis isomer, and also the "particle in a 3D box" model sets its importance here.

\section{Acknowledgment}

The author is indebted to the computer center at the Department of Chemistry of this College for providing computer facilities, and the author extends his thanks to Dr Saikat Sarkar, Department Of Chemistry, Santipur College, West Bengal, INDIA, for all of his encouragement.

\section{Disclosure}

The author reports no conflicts of interest in this work.

\section{References}

1. Cotton FA, Wilkinson G, Murillo CA, Bochmann M. Advanced Inorganic Chemistry. 6th ed. USA: John Wiley \& Sons Ltd; 2008:337.

2. Mouräo ZS, Melo A. Energy decomposition analysis of cis and trans isomers of 1,2dihaloethylenes and 2-butene. J Mol Str THEOCHEM. 2010;946(1-3):7-12.

3. Ismail N, Vairamani M, Rao R N. Determination of cis and trans isomers of monocrotophosin technical products reversed phase column liquid chromatography. J Chromatogr A. 2000;903(1-2):255-260.

4. Concepció J, Loeb B, Simón Manso Y, Zuloaga F. Influence of L-type ligand on the relative stability and inter conversion of cis-trans $\left[\mathrm{Ru}(\mathrm{Phen})_{2} \mathrm{~L}_{2}\right]^{\mathrm{n}+}$ type complexes $-\mathrm{a}$ theoretical study. Polyhedron. 2000;19(22-23):2297-2302.

5. Hush NS, Schambuger J, Bacskay GB. A quantum chemical computational study of the relative stabilities of cis and trans platinum dichloride in aqueous solution. Coord Chem Rev. 2005;249(3-4):299-311.

6. Dolgonos G. Relative stability and thermodynamic properties of $\mathrm{Si}_{2} \mathrm{H}_{4}$ isomers. Chem Phys Lett. 2008;466(1-3):11-15.

7. Alfaia CMM, Riberio PJLC, Trigo MJP, et al. Irradiation effect on fatty acid composition and conjugated linoleic acid isomers in frozen lamb meat. Meat Sci. 2007;77(4):689-695.

8. Senthilkumar K, Kolandaivel P. Molecular structure conformational stability and cis effect of 1,4-dichlorobutadiene - a quantum chemical study. J Mol Str THEOCHEM. 2002;577(1):69-79.

9. Kuznetsov ML, Hankka M, Pombeiro JL, Nazarov AA, Kukushkin VY. Theoretical study of the relative stability of isomeric forms of platinum carboxamide complexes. Inorganica Chim Acta. 2003;350:245-251.

10. Paul A, Bittermann H, Gmeiner P. Triazolopeptides: chiro specific synthesis and cis/trans prolyl ratios of structural isomers. Tetrahedron. 2006;62(38):8919-8927.

11. Kang YK. Cis-trans isomerization of N-acetyl- $\mathrm{N}$-methyl amides of 5-methyl praline and 5,5,-dimethylproline. J Mol Str THEOCHEM. 2002;585(1-3):209-221.

12. Dasgupta B, Chakraborti P, Basu G. Enhanced stability of cis pro-pro peptide bed in pro-pro-phe sequence motif. FEBS Lett. 2007;581(23): 4529-4532. 
13. Schieber A, Caele R. Occurrence of carotenoid isomers in food technological, analytical, and nutritional implications. Trends Food Sci Technol. 2005;16(9):416-422.

14. Geerlings P, De Proft F, Langenaeker W. Conceptual density functional theory. Chem Rev. 2003;103(5):1793-1873.

15. Chermette H. Chemical reactivity indexes in density functional theory. J Comput Chem. 1999;20(1):129-154.

16. Parr RG, Donelly RA, Levy M, Palke WE. Electronegativity: the density functional view point. J Chem Phys. 1978;68(18):3801-3807.

17. Jaque $P$, Toro-Labbé A. Characterization of copper cluster through the use of density functional theory reactivity descriptors. J Chem Phys. 2002;117(7):3208-3218.

18. Gutierrez-oliva S, Jaque P, Toro-Labbé A. Using Sanderson's principle to estimate global electronic properties and bond energies of hydrogenbonded complexes. J Phys Chem A. 2000;104(39):8955-8964.

19. Perez P, Toro-Labbé A. Characterization of keto enol tautomerism of acetyl derivatives from the analysis of energy: chemical potential and hardness. J Phys Chem A. 2000;104(7):1557-1562.

20. Toro-Labbé A. Characterization of chemical reactions from the profiles of energy, chemical potential and hardness. J Phys Chem A. 1999; 103(22):4398-4403.

21. Zhou Z, Parr RG. Activation hardness: new index for describing the orientation of electrophilic aromatic substitution. J Am Chem Soc.1990;112(15):5720-5724.

22. Pearson RG. Recent advances in the concept of hard and soft acids and bases. J Chem Educ. 1987;64(7):561-567.

23. Pearson RG. Maximum chemical and physical hardness. J Chem Educ. 1999;76(2):267-274.

24. Pearson RG. Electronic spectra and chemical reactivity. J Am Chem Soc. 1988;110(7):2092-2097.

25. Pearson RG. The principle of maximum hardness. Acc Chem Res. 1993; 26(5):250-255.

26. Gazquez JL. Bond energies and hardness differences. J Phys Chem A. 1997;101(49):9464-9469.

27. Sola M, Toro-Labbé A. The Hammond postulate and the principle of maximum hardness in some intramolecular rearrangement reactions. J Phys Chem A. 1999;103(44):8847-8852.

28. Datta D. On Pearson's HSAB principle Inorg Chem. 1992;31(13):2797.

29. Torrent-Sucarrat M, JLuis JM, M.Duran, Sola M. The hardness profile as a tool to detect spurious stationary points in the potential energy surface. J Chem Phys. 2004;120(23):10914-10924.

30. Parr RG, Chattaraj PK. Principle of maximum hardness. J Am Chem Soc. 1991;113(5):1854-1855.

31. Chattaraj PK, Lee H, Parr RG. HSAB principle. J Am Chem Soc. 1991;113(5):1855-1856.

32. Gutierrez-oliva S, Jaque P, Toro-Labbé A. Reviews in Modern Quantum Chemistry: A Celebration with Contributions of RG Parr and KD Sen. Singapore: World Scientific Press; 2002.

33. Torrent-Sucarrat M, Luis J, Duran M, Sola M. On the validity of the maximum hardness and minimum polarizability principles for nontotally symmetric vibrations. J Am Chem Soc. 2001;123(32): 7951-7952.

34. Morell C, Grand A, Toro-Labbé A. New dual descriptor for chemical reactivity. J Phys Chem A. 2005;109(1):205-212.

35. Ghosh DC, Jana J, Biswas R. Quantum chemical study of the umbrella inversion of the ammonia molecule. Int J Quantum Chem. 2000;80(1): $1-26$.

36. Ghosh DC, Jana J, Bhattacharyya S. Density functional and molecular orbital study of physical process of inversion of nitrogen trifluoride $\left(\mathrm{NF}_{3}\right.$ ) molecule. Int J Quantum Chem. 2002;87(3):111-134.
37. Ghosh DC. Density functional and frontier orbital study of the physical process of the conformational isomerism of ethane. J Ind Chem Soc. 2002;79(3):240-248.

38. Nguyen TN, De Proft F, Nguyen MT, Geerlings P. Theoretical study of [2+1] cycloaddition of CO and CS to acetylenes forming cyclopropenones and cyclopropenethiones. J Org Chem. 2001;66(12): 4316-4326.

39. Nguyen LT, De Proft F, Nguyen MT, Geerlings P. Theoretical study of cyclopropenones and cyclopropenethiones: principles decomposition via intermediates. J Chem Soc Perkin 1. 2001;2(6):898-905.

40. Chandra AK, Nguyen MT. Density functional approach to regiochemistry, activation energy, and hardness profile in 1,3-dipolar cycloadditions. J Phys Chem A. 1998;102(30):6181-6185.

41. Cardens-Jiron GI, Gutierrez-oliva S, Melin J, Toro-Labbé A. Relations between potential energy, electronic chemical potential, and hardness profiles. J Phys Chem A. 1997;101(25):4621-4627.

42. Cardens-Jiron GI, Letelier JR, Toro-Labbé A. The internal rotation of hydrogen thioperoxide: energy, chemical potential, and hardness profiles. J Phys Chem A. 1998;102(40):7864-7871.

43. Ghanty TK, Ghosh SK. Molecular hardness, polarizability, and valency variation of formamide and thioformamide on internal rotation: a density functional study. J Phys Chem A. 2000;104(13):2975-2979.

44. Uchimaru T, Chandra AK, Kawahara S, Matsumura K, Tsuzuki S, Mikami M. Internal bond rotation in substituted methyl radicals, $\mathrm{H}_{2} \mathrm{~B}-\mathrm{CH}_{2}, \mathrm{H}_{3} \mathrm{C}-\mathrm{CH}_{2}, \mathrm{H}_{2} \mathrm{~N}-\mathrm{CH}_{2}$ and $\mathrm{HO}-\mathrm{CH}_{2}$ : hardness profiles. J Phys Chem A. 200;105(8):1343-1353.

45. Nguyen MT, Chandra AK, Sakai S, Morokuma K. Another look at the mechanism of the concerted 1,3-dipolar cycloaddition of fulminic acid to acetylene. J Org Chem. 1999;64(1):65-69.

46. Kar T, Scheiner S, Sannigrahi AB. Ab initio calculations of hardness and chemical potential of open shell systems using SCF, MP2 and MP4 methods. J Mol Struct THEOCHEM. 1998;427(1-3):79-85.

47. Roy RK, Chandra AK, Pal S. Correlation of polarizability, hardness and electronegativity: polyatomic molecules. J Phys Chem. 1994;98(41):10447-10450.

48. Martin RB. Localized and spectroscopic orbitals: Squirrel ears on water. J Chem Educ. 1988;65(8):668-670.

49. Pople JA, Beveridge DL. Approximate Molecular Orbital Theory. New York: Mc Graw-Hill; 1970.

50. Trindle C, Sinanoğlu O. Semiempirical method for the determination of localized orbitals in molecules. J Chem Phys. 1968;49(1):65-71.

51. Fischer H, Kollmar H. Energy partitioning with the CNDO method. Theor Chim Acta. 1970;6(3):163-174.

52. Geerlings P, De Proft F, Langenaeker W. Density Functional Theory: A Bridge between Chemistry and Physics. VUB University Press: 1998.

53. Parr RG, Mortier WJ. The use of global and local molecular parameters for the analysis of the gas phase basicity of amines. J Am Chem Soc. 1986;108(19):5708-5711.

54. Koopmans T. Ordering of wave functions and eigenenergies to the individual electrons of an atom. Physica. 1933;1(1-6):104-113.

55. Pople JA, Santry DP, Segal GA. Approximate self-consistent molecular orbital theory. I. invariant procedures. J Chem Phys. 1965; 43(10):S129.

56. Gordon MS. Molecular orbital study of internal rotation. J Am Chem Soc. 1969;91(12):3122-3140.

57. Edmiston C, Reudenberg K. Localized atomic and molecular orbitals Rev Mod Phys. 1963;35(3):457-464.

58. Reudenberg K. The physical nature of the chemical bond. Rev Mod Phys. 1962;34(2):326-376. 
Reports in Theoretical Chemistry

Dovepress

\section{Publish your work in this journal}

Reports in Theoretical Chemistry is an international, peer-reviewed, open access journal publishing original research, reports, reviews and commentaries on all areas of theoretical chemistry. The manuscript management system is completely online and includes a very quick and fair

peer-review system. Visit http://www.dovepress.com/testimonials.php to read real quotes from published authors. 